(REVIEW ARTICLE)

\title{
Antioxidants outlook: Diseases preventive versus curative/therapeutic impact
}

\author{
Alrawi Rakhad 1, ${ }^{*}$, Abdulrahman Jubran Mohammed 2, Al-Rawi Rafal A ${ }^{3}$ and Al-Rawi Rafat 4 \\ ${ }^{1}$ Grand Canyon University and Arizona College. Arizona, USA. \\ ${ }^{2}$ Center of Research and Educational Studies, Ministry of Education, Iraq. \\ ${ }^{3}$ Department of Pathology, Hawler Medical University, Erbil, Kurdistan, Iraq. \\ ${ }^{4}$ Arlöv Kronetorpsvägen 2 (Burlöv Center), Malmo, Sweden.
}

Publication history: Received on 05 June 2020; revised on 15 June 2020; accepted on 18 June 2020

Article DOI: https://doi.org/10.30574/wjarr.2020.6.3.0189

\begin{abstract}
Antioxidants are natural chemical substances that exist in foods such as vitamins, minerals and other compounds that are believed to help preventing disease by neutralize free radicals. Free radical, are formed by normal body metabolic processes, and by exposure to environmental contaminants like pollution and cigarette smoking. Without adequate amounts of antioxidants, these free radicals travel throughout the body causing cells damage and homeostatic disruption. Part of this cellular damage leads to one of several diseases among them are coronary heart disease (atherosclerosis as well as cardiovascular), and cancer.
\end{abstract}

Keywords: Antioxidants; Free radical; Disease prevention and therapy

\section{Background}

\subsection{Antioxidants}

Antioxidants are chemical substances that interact with free radicals, thus preventing or slow cells damage and consequently boost the overall health. Antioxidants are considered free radical scavengers, as they neutralize these free radicals. The sources of antioxidants can be natural (certain plant foods), or artificial (drugs and supplements). The body produces some antioxidants, known as endogenous antioxidants. On the other hands, antioxidants that come from outside the body are called external or exogenous sources, primarily from foods. These exogenous antioxidants are called dietary antioxidants. The best source(s) of antioxidants are green and red vegetables, legumes, fruits, nuts, papaya, seeds, and whole grains. Furthermore, some other good sources are brown rice, oatmeal, soybeans, sweet potatoes, and wheat germ. Dietary antioxidants that come from outside the body include vitamin A, vitamin C, vitamin E, beta-carotene, lutein, lycopene, catechins, flavonoids, flavones, polyphenols, selenium, manganese, zeaxanthin, copper, iron, and zinc. All these antioxidants are found in plant foods. Each antioxidant serves a different function and may interacting with another. This is why it is important to have a varied diet.

Antioxidants play important roles in the maintenance of cellular integrity and thus are critical in maintaining the homeostasis of the host immune system. A balance between the levels of pro-oxidants and antioxidants defines the cellular fate of genomic integrity via maintaining the redox status of the cells. An aberration in this balance modulates host immunity that affects normal cellular signaling pathways resulting in uncontrolled proliferation of cells leading to neocarcinogenesis. For decades, there have been scientific debates on the use of antioxidants for the treatment of human cancers. Antioxidants can play role in protection against cells damage that free radicals cause, known as oxidative stress. The activities and processes that can lead to oxidative stress include: mitochondrial activity, excessive exercise, tissue trauma, inflammation and injury, consumption of certain foods, especially refined and processed foods, fats, artificial sweeteners, , smoking, environmental pollution, radiation, exposure to chemicals, such as pesticides and drugs

\footnotetext{
${ }^{*}$ Corresponding author: Alrawi Rakhad
} 
including chemotherapy. Such activities and exposures can resulted in cells damage, which consequently leads to an increase in enzymes that generate free radicals, and a disruption of electron transport chains, all these physiological changes resulted oxidative stress, which has been linked to heart disease, cancer, arthritis, atherosclerosis, respiratory diseases, immune deficiency, vision loss and other abnormal or inflammatory conditions. Intake of antioxidants reduces these risks of these diseases, through their action to neutralize and scavenger free radicals. Foods have many substances that can act as antioxidant; each has its own role and can interact with each other to help the body to work effectively [1-3].

\section{Free radicals and Oxidative stress}

Free radicals are highly reactive chemicals that are formed naturally in the body and play an important role in many normal cellular processes. A balance between antioxidants and free radicals is necessary for proper physiological function. If free radicals surpass the body's ability to regulate them, an oxidative stress occurs. At high concentrations, however, free radicals can have the potential to harm cells as well as to be hazardous to damage all major components of cells, including DNA, proteins, and cell membranes causing a number of human diseases and may play a role in the development of cancer and other health conditions. Abnormally high concentrations of free radicals in the body can be caused by several factors that may be internal, such as inflammation, or external, such as pollution, UV exposure, some metals, and cigarette smoke. Free radicals that contain the element oxygen are the most common type of free radicals produced in living tissue, which have a tendency to donate oxygen to other substances, thus it termed "reactive oxygen species," or "ROS". Free radicals are waste substances produced by cells as by-products of metabolic process and/or due to react to the environmental toxins. All cells contain mitochondria, which combine oxygen and glucose to produce energy, carbon dioxide, and water. Free radicals produced as by-products of this metabolic process. External environmental factors, such as cigarette smoke, pesticides, and ozone, can also cause the formation of free radicals in the body. If the body cannot process and remove free radicals efficiently, oxidative stress can be resulted. Free radicals include reactive oxygen species, and superoxide hydroxyl radical, and nitric oxide radical. Nevertheless, the ROS is used to fight against extracellular pathogens, including viral and bacterial infections, causing alterations in signaling pathways and neocarcinogenesis. It is well documented that high environmental temperature challenges the animal ability to maintain thermal, hormonal, water, and mineral balance. Heat stress stimulates production of free radicals (superoxide anion radicals, hydroxyl radical, hydrogen peroxide and singlet oxygen) which are continuously produced in normal metabolism [4 -6].

\section{Natural dietary versus synthetic antioxidants}

Results of several studies showed conflicting findings, in regards to using antioxidants. Some found benefit in using antioxidants. Some did not. Still others found potential harm from the antioxidants as supplements. Application of external source of antioxidants can assist in coping oxidative stress. Synthetic antioxidants have been reported to be dangerous for human health. Research for effective, nontoxic natural compounds with anti- oxidative activity has been intensified in recent years. The National Institutes of Health $(\mathrm{NIH})$ warn that high doses of antioxidant supplements can be harmful. A high intake of beta-carotene, for example, has been linked to an increased risk of lung cancer in smokers. A high dose of vitamin $\mathrm{E}$ has been found to increase the risk of prostate cancer, and the use of some antioxidant supplements has been linked to a greater risk of tumor growth. Antioxidant supplements may also interact with some medications. Furthermore, Free radicals have been linked to a range of diseases, including heart disease, cancer, and vision loss, but this does not mean that an increased intake of antioxidants will prevent these diseases. Antioxidants from artificial sources may increase the risk of some health problems. As a result, it is important to seek out natural sources of antioxidants, in the form of a healthful diet. Consuming fruits and vegetables has been linked to a lower rate of chronic diseases, and antioxidants may play a role. However, it is unlikely that consuming added antioxidants, especially in processed foods, will provide significant benefits. Studies showed that antioxidants may influence the response to chemotherapy and its side effects. The result indicated that some chemo-drugs induce oxidative stress and lead to normal tissue apoptosis and the entry of cancer cells to a dormant G0 state. Through the suppression of oxidative stress, antioxidants protected normal cells and bring the tumor out of dormancy to expose it to chemotherapies. The study of "Prestigious British medical journal, the Lancet (2020) indicated that Vitamin E supplement did not significantly provide any benefit in decreasing the risk of cardiovascular stroke or death. They concluded that diet supplements are no substitute for good eating habits, exercise, weight loss and smoking cessation as a means of minimizing the risk of heart disease. Many reports recommended foods (such as fruits, vegetables and whole grains) that are sources of natural antioxidants. Other benefits can be obtained from food such as vitamins, minerals, flavonoids and lycopenes that are linked to reduced risk of many diseases and are not included in vitamin supplements. Currently, recommendations focus more on the naturally-occurring antioxidants found in natural dietary foods. Reports indicated that dietary antioxidants most often refer to those found in fruits, vegetables, and other foods (nuts, oils, and seeds). 
The most common dietary antioxidants include vitamin A, vitamin C (ascorbic acid), vitamin E (alpha and gamma tocopherol), beta-carotene and related carotenoids, lutein, lycopene, selenium, manganese, zeaxanthin, catechins, flavonoids, flavones, polyphenols, and phytoestrogens. All these antioxidants are found in plant foods. Each antioxidant serves a different function and may interacting with another. This is why it is important to have a varied diet. The best source(s) of these antioxidants are green and red vegetables, legumes, fruits, nuts, papaya, seeds, and whole grains. Furthermore, some other good sources are brown rice, oatmeal, soybeans, sweet potatoes, and wheat germ. The following foods are best sources of particular antioxidants: dairy products, eggs, carrots, and liver (rich in Vitamin A); berries, oranges, pomegranateand green peppers (rich in vitamin C); nuts, seeds, plant crops oils, and green leafy vegetables (rich invitamin E); carrots, peas, spinach, and mango(Beta-carotene); tomatoes and watermelon (Lycopene); Green leafy vegetables, corn, papaya, and oranges (Lutein); and rice, corn, wheat, oats, rye, nuts, eggs, cheese, and legumes (Selenium). Other foods that are believed to be good sources of antioxidants include eggplants, kidney beans, green and black teas, red grapes, and dark chocolate. Furthermore, the following foods are good sources of antioxidants blueberries, apples, broccoli, spinach, and lentils [12 - 14].

On the other hand, results carried out in laying hens suggested that antioxidant supplementation attenuates oxidative stress response. These data support the hypothesis that supplemental antioxidants improve hen well-being by reducing heat stress associated physical and physiological damage [15].

Furthermore, result of the role of antioxidants in maintenance of cellular integrity and the homeostasis of the host immune system that prevent uncontrolled proliferation of cells (neocarcinogenesis) [16].

\section{Antioxidants and Immunity}

Natural antioxidants may be defined as molecules that prevent cell damage against free radicals and are critical for maintaining optimum health in both animals and humans. In all living systems, cells require adequate levels of antioxidant defenses in order to avoid the harmful effect of an excessive production of reactive oxygen species (ROS) and to prevent damage to the immune cells. Antioxidants are absolutely necessary to regulate the reactions that release free radicals. Antioxidant nutrients commonly included in the diet such as vitamin E, vitamin C, $\beta$-carotene, selenium, copper, iron and zinc improve different immune function exhibiting an important protective role in infections caused by bacteria, viruses or parasites. As a result, dietary antioxidants have been related to modulate the host susceptibility or resistance to infectious pathogens [17]. Recently progress regarding the potential benefit of dietary antioxidants in the treatment of chronic diseases with a special focus on immune system and neurodegenerative disorders has been investigated. It is well established that reactive oxygen species (ROS) play an important role in the etiology of numerous diseases, such as atherosclerosis, diabetes and cancer. Among the physiological defense system of the cell, the relevance of antioxidant molecules, such as glutathione and vitamins is quite well established. The potential therapeutic applications of antioxidants in free radical-related diseases led to the hypothesis of their use to slow down or reverse, for example, symptoms associated with neurodegenerative disorders, such as Alzheimer's disease (AD), and Parkinson's disease (PD). Such effect could occur through a block of proinflammatory cytokines action and the resulting oxidative damage [18 - 20]. There are various essential micronutrients including minerals and vitamins in the diet, which play a vital role in reinforcing antioxidant performance, affecting the genes and encoding proteins for carcinogenesis. Many of these antioxidant agents are available as dietary supplements and are predominant worldwide. However, the best antioxidant micronutrient (or a combination of micronutrients) for reducing cancer risks is unknown. The development of cancer has been linked to an inability of the host immune system to respond appropriately to tumor antigens, which leads to tumor immune evasion. The recognition and eradication of cancer cells by the immune system are categorized as elimination, equilibrium, and escape phases [21]. A review focused on the implications of antioxidant use as adjuncts in cancer therapy with an emphasis on immunomodulation and radiosensitization was indicated that antioxidants play important roles in the maintenance of cellular integrity and thus are critical in maintaining the homeostasis of the host immune system. A balance between the levels of pro-oxidants and antioxidants defines the cellular fate of genomic integrity via maintaining the redox status of the cells. An aberration in this balance modulates host immunity that affects normal cellular signaling pathways resulting in uncontrolled proliferation of cells leading to neocarcinogenesis. To date, findings of several studies announced conflicting results, in regards to using antioxidants for the treatment of diseases. The results differed from one another. Some found benefit in using antioxidants. Some didn't. Still others found potential harm from the antioxidants as supplements. Nevertheless, the involvement of the reactive oxygen species (ROS) is well documented in various disease processes, including cancers $[16,22,23]$. 


\section{Conclusion}

It was concluded that antioxidants play important roles in the maintenance of cellular integrity and thus are critical in maintaining the homeostasis of the immune system and for maintaining optimum health. Natural antioxidants foods are important strategy to boost immune system and consequently preventing many diseases.

\section{Compliance with ethical standards}

\section{Acknowledgments}

The authors express their appreciation to Professor Abdulhameed, Abdulrazaq for critical review of this article.

\section{Disclosure of conflict of interest}

The authors declare that they have no competing interests.

\section{References}

[1] Yang R and Liu Y. (2017). Structure, Function, and Nutrition of Selenium-Containing Proteins from Foodstuffs. In: Zhao G. (eds) Mineral Containing Proteins. Springer, Singapore

[2] EvangelosZoidis, IsidorosSeremelis, NikolaosKontopoulos, and Georgios P. (2018). Danezis. SeleniumDependent Antioxidant Enzymes: Actions and Properties of Selenoproteins. Antioxidants (Basel), 7(5), 66.

[3] Cleveland Clinic medical professional. (2019). Cleveland, Ohio 44195.

[4] Lobo V, A. Patil, A. Phatak and N. (2010). Chandra. Free radicals, antioxidants and functional foods: Impact on human health. Pharmacogn Rev, 4(8), 118-126.

[5] Jamie Eske. (2019). Health line Media UK Ltd. Medical News Today. Newsletter.

[6] Bernabucci U, Ronchi B, Lacetera N andNardone A. (2002). Markers of oxidative status in plasma and erythrocytes of transition dairy cows during hot season. J Dairy Sci, 85(9), 2173-9.

[7] Satoh H, Moriguchi, T and Taguchi K. (2010). Nrf2-deficiency creates a responsive microenvironment for metastasis to the lung. Carcinogenesis,31, 1833-1843.

[8] Elmarakby AA, DM Pollock and JDImig. (2007). Therapeutic Areas I: Central Nervous System, Pain, Metabolic Syndrome, Urology, Gastrointestinal and Cardiovascular. V. 6. Comprehensive Medicinal Chemistry II: Editor: Michael Williams. Comprehensive Medicinal Chemistry II.

[9] Paiva, CN andBozza, MT. (2014). Are reactive oxygen species always detrimental to pathogens? Antioxid Redox Signal, 20, 1000-1037.

[10] Cui J, Chen Y, Wang, HY and Wang RF. (2014). Mechanisms and pathways of innate immune activation and regulation in health and cancer. Hum VaccinImmunother, 10, 3270-3285.

[11] Bernardes SS, de Souza-Neto FP andRamalho, LN. (2015). Systemic oxidative profile after tumor removal and the tumor microenvironment in melanoma patients. Cancer Lett, 361, 226-232.

[12] Forman HJ, Davies KJ andUrsini F. (2014). How do nutritional antioxidants really work: nucleophilic tone and para-hormesis versus free radical scavenging in vivo. Free RadicBiol Med, 66, 24.

[13] Lee IM, Cook NR, Gaziano JM, et al. (2005). Vitamin E in the primary prevention of cardiovascular disease and cancer: the Women's Health Study: a randomized controlled trial. JAMA,294, 56.

[14] El-Sabban F. (2014). The antioxidant advantage of the Mediterranean diet in cardiovascular disease. Nutrition and Dietary Supplements, 6, 35-40.

[15] Roghayeh I, Abolfazl B, Mohammad RM, Didier L, Michel C and Graciela P. (2020). The conundrum of dietary antioxidants in cancer chemotherapy. Nutrition Reviews, 78(1), 65-76.

[16] Anita Thyagarajan and Ravi PSahu. (2018). Potential Contributions of Antioxidants to Cancer Therapy: Immunomodulation and Radiosensitization Integrative Cancer Therapies, 17(2), 210-216. 
[17] Puertollano MA, Puertollano E, de Cienfuegos GÁ and de Pablo MA. (2011). Dietary antioxidants: immunity and host defense. Curr Top Med Chem, 11(14), 1752-66.

[18] Daria B, Cesare M, Mariagrazia RS, Paolo B, Giuseppina C, Laurence L et al. (2008). The role of antioxidant supplement in immune system, neoplastic, and neurodegenerative disorders: a point of view for an assessment of the risk/benefit profile. Nutrition,7(29).

[19] Mancuso C, Bates TE, Butterfield DA, Calafato S, Cornelius C, De Lorenzo A, DinkovaKostova AT and Calabrese V. (2007). Natural antioxidants in Alzheimer's disease. Expert OpinInvestig Drugs, 16(12), 1921-1931.

[20] Whitton PS. (2007). Inflammation as a causative factor in the aetiology of Parkinson's disease. Br J Pharmacol, $150(8), 963-976$.

[21] Xiayu W, Jiaoni C and Xu W. (2017). Dietary Antioxidants: Potential Anticancer Agents. Nutrition and Cancer, 69(4), 521-533.

[22] Sreevalsan S and Safe S. (2013). Reactive oxygen species and colorectal cancer. Curr Colorectal Cancer Rep,9, 350-357.

[23] Marinescu S, Anghel R, Gruia MI and Beuran M. (2014). Involvement of reactive oxygen species in the mechanisms associated with cervical cancer specific treatment. Chirurgia (Bucur), 109, 806-811.

\section{How to cite this article}

Alrawi R, Abdulrahman JM, Al-Rawi RA and Alrawi RA. (2020). Antioxidents outlook: Diseases preventive versus curative/therapeutic impact. World Journal of Advanced Research and Reviews, 6(3), 175-179. 